\title{
Os desafios na aplicabilidade da educação interprofissional no âmbito da atenção primária em saúde
}

Challenges in the applicability of interprofessional education in the context of primary health care Desafíos en la aplicabilidad de la educación interprofesional em el contexto de la atención primaria de salud

Maria Letícia Cardoso da Silva Barbosa ORCID: https://orcid.org/0000-0002-2935-6882 Universidade Federal de Campina Grande, Brasil E-mail: marialeticia20151@ @otmail.com

Maria Eduarda Wanderley de Barros Silva ORCID: https://orcid.org/0000-0002-4642-3282 Universidade Federal de Campina Grande, Brasil E-mail: eduarda.wanderley@outlook.com

Anna Thereza Leite Cavalcanti ORCID: https://orcid.org/0000-0002-0313-3525 Universidade Potiguar, Brasil E-mail: annatherezalc@gmail.com Iara Maria Lins Cury ORCID: https://orcid.org/0000-0002-2499-0921 Universidade Potiguar, Brasil E-mail: iaracury_@hotmail.com

Pármenas Augusto Vasconcelos dos Santos ORCID: https://orcid.org/0000-0001-8484-5403 Universidade Potiguar, Brasil E-mail: parmenasaugusto@hotmail.com

Milena Ratacasso Coimbra ORCID: https://orcid.org/0000-0002-6219-5953 Universidade Federal de Pernambuco, Brasil E-mail: ratacasso@gmail.com

Nayhara Rayanna Gomes da Silva ORCID: https://orcid.org/0000-0002-3162-6079

Universidade Federal de Pernambuco, Brasil E-mail: nayhara.gomes@ufpe.br

Rebeka Ferreira Coelho

ORCID: https://orcid.org/0000-0002-8063-7225

Universidade Federal de Pernambuco, Brasil E-mail: rebeka.coelho@ufpe.br

Maria Luiza Menezes Monteiro Costa ORCID: https://orcid.org/0000-0002-2499-0921 Universidade Potiguar, Brasil

E-mail: mlmenezesmc@gmail.com

Deoclecio Neto Mendes Diniz ORCID: https://orcid.org/0000-0002-3060-4660 Universidade Potiguar, Brasil

E-mail: deoclecio2303@gmail.com

João Victor Araújo Martins ORCID: https://orcid.org/0000-0002-5855-8344 Universidade Potiguar, Brasil

E-mail: jvictoramartins1999@gmail.com

Maria Eduarda de Lima Onório ORCID: https://orcid.org/0000-0002-4701-2282 Escola de Ensino Superior do Agreste Paraibano, Brasil

Email: m.eduardaonorio.enf@gmail.com

Yago Mavignier Amaral da Costa ORCID: https://orcid.org/0000-0001-8406-0745 Universidade Potiguar, Brasil

Email: yagomavignier@hotmail.com 


\author{
Tayron Felipe da Silva \\ ORCID: https://orcid.org/0000-0002-4135-6471 \\ Universidade Potiguar, Brasil \\ Email: tayronfelipe@ outlook.com \\ Larissa Lima Soares \\ ORCID: https://orcid.org/0000-0002-7682-372X \\ Centro Universitário Tiradentes, Brasil \\ E-mail: larissalyma@hotmail.com
}

\title{
Resumo
}

O estudo possui como objetivo identificar os principais desafios na aplicabilidade da educação interprofissional no âmbito da Atenção Primária em Saúde. Trata-se de uma revisão integrativa da literatura de abordagem qualitativa, norteada pela seguinte questão de pesquisa "Quais as principais dificuldades encontradas na aplicabilidade da educação interprofissional no âmbito da Atenção Primária de Saúde?”. A coleta de dados ocorreu entre os meses de setembro e outubro a partir da base de dados da Literatura Latino-Americana e do Caribe e Ciências da Saúde, Bases de Dados em Enfermagem e Scientific Electronic Library Online. Para a estratégia de busca foram utilizados os descritores em Ciências da Saúde (DECS), sendo "Educação Interprofissional", "Atenção Primária à Saúde" e "Equipe de Assistência ao Paciente". Após o processo de elegibilidade dos estudos, foi propicio a leitura na íntegra dos 8 materiais. Os profissionais de saúde do NASF revelam que o processo de formação na graduação possui um déficit de conteúdos e práticas referentes à atuação generalista na atenção básica com algumas variações de um curso para outro ou entre universidades. Isso revela que há uma necessidade de ações e encontros de educação permanente no cotidiano de trabalho. Assim, mediante a todos os desafios o desenvolvimento da educação interprofissional e interdisciplinar só será consolidada for ampliada, disseminada e potencializada experiência educacionais para que se tenha interações entre as variadas áreas para que seja visto o impacto dessas ações na Atenção Básica de Saúde.

Palavras-chave: Educação interprofissional; Atenção primária à saúde; Equipe de assistência ao paciente.

\begin{abstract}
The study aims to identify the main challenges in the applicability of interprofessional education in the context of Primary Health Care. It is an integrative literature review with a qualitative approach, guided by the following research question "What are the main difficulties encountered in the applicability of interprofessional education in the context of Primary Health Care?". Data collection took place between September and October in the Latin American and Caribbean Literature and Health Sciences, Databases in Nursing and Scientific Electronic Library Online databases. For the search strategy, the descriptors in Health Sciences (DECS) were used, being "Interprofessional Education", "Primary Health Care" and "Patient Assistance Team". After the study eligibility process, it was possible to read the 8 materials in their entirety. The NASF health professionals reveal that the graduation training process has a deficit of content and practice regarding the general role in primary care, with variations from one course to another or between universities. This reveals the need for permanent education actions and meetings in daily work. Thus, given all the challenges, the development of interprofessional and interdisciplinary education will only be consolidated by the expanded, disseminated and improved educational experience so that interactions between different areas can be perceived to see the impact of these actions on Primary Health Care.
\end{abstract}

Keywords: Interprofessional education; Primary health care; Patient care team.

\section{Resumen}

El estudio tiene como objetivo identificar los principales desafíos en la aplicabilidad de la educación interprofesional en el ámbito de la Atención Primaria en Salud. Se trata de una revisión integradora de la literatura de enfoque cualitativo, guiada por la siguiente cuestión de investigación "¿Cuáles son las principales dificultades encontradas en la aplicabilidad de la educación interprofesional en el ámbito de la Atención Primaria de Salud?". La recolección de datos tuvo lugar entre los meses de septiembre y octubre a partir de la base de datos de la Literatura Latinoamericana y del Caribe y Ciencias de la Salud, Bases de Datos en Enfermería y Scientific Electronic Library Online. Para la estrategia de búsqueda fueron utilizados los descriptores por el Descriptores en Ciencias de la Salud (DECS), siendo "Educación Interprofesional", "Atención Primaria a la Salud" y "Equipo de Atención al Paciente". Tras el proceso de elegibilidad de los estudios, fue propicio la lectura en su totalidad de los 8 materiales. Los profesionales de salud del NASF revelan que el proceso de formación en la graduación posee un déficit de contenidos y prácticas referentes a la actuación generalista en la atención básica con algunas variaciones de un curso para otro o entre universidades. Esto revela que hay una necesidad de acciones y encuentros de educación permanente en el cotidiano de trabajo. Así, a través de todos los desafíos el desarrollo de la educación interprofesional e interdisciplinaria solo será consolidada y ampliada, Diseminada y potenciada experiencia educativa para que se tengan interacciones entre las variadas áreas para que se vea el impacto de esas acciones en la Atención Básica de Salud.

Palabras clave: Educación interprofesional; Atención primaria de salud; Equipo de atención al paciente. 


\section{Introdução}

Após a revolução industrial e com a evolução histórica de produção de conhecimentos, foi cada vez mais evoluindo sobre as condições de saúde e qualidade de vida da população. Com isso, desde a década de 1970 procura-se compreender os fatores responsáveis pelo processo de saúde-doença, por isso alguns cientistas e autores propuseram modelos de aprendizagem para os determinantes de saúde sendo o mais aceito atualmente no Brasil, o Modelo de Determinantes Sociais de Dahlgren e Whitehead, que determinam os fatores do processo saúde-doença em vários níveis, indo do individual ao macrossocial (Ceballos, 2015).

Mediante as transformações do meio, encontram-se no diálogo entre os diversos campos de conhecimentos, ferramentas para abordar integralmente a complexidade dos problemas atuais de saúde, sendo interprofissionalismo um dos seus pilares. Desse modo, o interprofissionalismo possui uma definição de comunicação entre os diversos profissionais da área para que se tenha a integração de um cuidado especializado, intencionalmente estabelecendo vínculos e nexos entre si para alcançar conhecimentos e decisões conjuntas mais abrangentes diante de um mesmo objetivo de trabalho, opondo-se ao reducionismo e fragmentação (Santana\& Rossit, 2017).

A intenção principal da Atenção Básica no Brasil, é que sirva como porta de entrada do Sistema Único de Saúde, e como eixo estruturante de sua organização, seu funcionamento é regulado de acordo com a Política Nacional da Atenção Básica (PNAB). Essa normativa prevê o trabalho de uma equipe multiprofissional, das equipes de Saúde da Família (eSF), para que se tenha o atendimento à população de um território adscrito. Nessa perspectiva, o processo de trabalho em saúde sofre diversas transformações ao longo da história que, consequentemente, demandam que os profissionais de saúde envolvidos questionem suas práticas e perspectivas atuações (Peduzzi, 2016).

A formação multiprofissional se tornou um espaço intercessor para a equipe de saúde, pois propõe o encontro entre diversos usuários, docentes e profissionais do serviço com a construção de relações e interações (Silva, Peduzzi, Orchard \& Leonello, 2015). Mesmo assim, é importante ressaltar que frequentemente na Atenção Primária a Saúde (APS), o trabalho da equipe multiprofissional se apresenta com uma postura de espera pelo sujeito das mudanças, ou seja, com as fragilidades que o SUS carrega a frustração dos profissionais se tornam cada vez mais realística (Cardoso \& Hennington, 2011).

A incorporação da interprofissionalidade na atenção à saúde se faz necessária para a resposta de alguns aspectos da realidade de produção dos serviços que se configura por forte fragmentação do trabalho em saúde, exposição do paciente a atos suplicados além de maiores possibilidades de erros de diagnóstico e tratamento. Um dos pontos divergentes que contribuem para os desafios enfrentados no sistema de trabalho refere-se aos profissionais de saúde por trabalharem juntos, entretanto serem formados separadamente (Costa, Filho, Brandão \& Silva, 2018).

Nesse contexto, existe uma crescente incorporação da Educação Interprofissional (EIP), como um processo formativo e de trabalho em saúde em todo o mundo, com a crença de que a implementação de seus princípios poderia melhorar a satisfação no trabalho, melhorar a resolutividade das demandas e aumento a apreciação da comunidade sobre a equipe de saúde. Dessa forma, com a perspectiva da EIP, todos os profissionais de saúde deveriam participar ativamente em sistemas de saúde centrados assim na população e no usuário respeitando as diversas práticas de acordo com cada profissão, com análise crítica, ética e ênfase em práticas colaborativas (Rossit, Freitas, Batista \& Batista, 2018).

A interprofissionalidade permite subsidiar as práticas em equipe favorecendo a troca de conhecimentos, a colaboração nas atividades exercidas, a atenção corresponsável às necessidades em saúde para a construção de projetos terapêuticos e de promoção da saúde, além do agir coletivo em território e desenvolvimento de vínculos mais intensos com a equipe.A interprofissionalidade remete a qualidade da atenção como resultado de um trabalho centrado no usuário/comunidade, mas pouco traz as barreiras encontradas pelos profissionais e o suporte direcionado aos trabalhadores e ao trabalho orientado às 
redes integradas de atenção, bem como ações que favoreçam planejamento e avaliação sob a integralidade, humanização e educação permanente em saúde (Ceccim, 2018).

A composição de equipes interdisciplinares e interprofissionais não é um modelo de resolução definitiva do conjunto de tensões, mas, é uma perspectiva que tem como pressupostos: a superação da fragmentação do trabalho e da individualização biomédica; a busca de reconstituição da integralidade do trabalho coletivo em saúde; e a qualificação do conjunto dos profissionais sob esses signos que objetiva democratizar o contexto do trabalho e efetivar integralmente o cuidado (Peduzzi, Agreli, Silva \& Souza, 2018).

O trabalho em equipe interprofissional compreende elementos do contexto social, político e econômico. Tem como propósito ao invés de reforçar a expectativa de autonomia e independência de cada profissão, na prática colaborativa os profissionais buscam diminuir a competição e substituir o desequilíbrio nas relações de poder no cuidado em saúde por relações de parceria interprofissional e responsabilidade coletiva (Peduzzi \& Agreli, 2018). É necessário conhecer como se organiza o trabalho interprofissional, no âmbito da atenção primária em saúde e suas demandas e competências na assistência (Previato \& Baldissera, 2017).

A colaboração como forma de trabalho interprofissional na atenção primária em saúde, pode ser entendida em duas modalidades, que são modificáveis a depender das necessidades ou condições dos usuários. A primeira é determinada como colaboração em equipe, onde os profissionais procuram alternativas entre os próprios membros da equipe ou entre equipes de uma mesma unidade básica de saúde, para avançar a qualidade da assistência à saúde e estimular a participação dos usuários no autocuidado. A segunda refere á colaboração em rede e com a população/comunidade, onde os profissionais da equipe buscam por alternativas da equipe e demais serviços, setores e com indivíduo, comunidade e família (Peduzzi \& Agreli, 2018).

Com isso, ainda se tem muito a avançar na prática e educação interprofissional sendo necessário o apoio de diversos atores sociais como Instituições de Ensino Superior (IES) e a Educação Profissional para que as políticas de saúde e de educação incorporem EIP e prática interprofissional em conjunto de mudanças propostas (Bourgeault, Grignon \& Grignon, 2013).

Portanto, a integração do ensino-serviço-comunidade é de extrema importância para minimizas os desafios enfrentados no desenvolvimento de uma prática integral e pautada na inteprofissionalidade. Assim, o estudo possui como objetivo identificar os principais desafios na aplicabilidade da educação interprofissional no âmbito da Atenção Primária em Saúde (APS). Considera-se imprescindivelmente o trabalho colaborativo em saúde, a comunicação e a equipe interprofissional como ferramentas que auxiliam na aplicabilidade da interprofissionalidade assegurando a qualidade de atenção em saúde nos serviços públicos, principalmente direcionados a atenção primária. No cenário nacional, a EIP ainda é incipiente e os resultados de sua implementação são pouco conhecidos, sendo salutar compreender as percepções de docentes, trabalhadores e estudantes sobre a articulação da EIP com as práticas de saúde na APS.

\section{Metodologia}

Trata-se de uma revisão integrativa da literatura de abordagem qualitativa (Ludke \& André, 2013). Sendo um processo de análise, busca e descrição de um corpo de conhecimento em busca de uma resposta específica, a revisão integrativa de literatura consiste em 6 etapas: 1) Identificação do tema e seleção da hipótese ou questão da pesquisa para elaboração da revisão, 2) Estabelecimento de critérios de inclusão e exclusão e busca na literatura, 3) categorização dos estudos, 4) avaliação dos estudos que serão incluídos na revisão, 5) interpretação dos resultados e 6) Síntese da revisão (Mendes, Silveira \& Galvão, 2008). Norteada pela seguinte questão de pesquisa "Quais as principais dificuldades encontradas na aplicabilidade da educação interprofissional no âmbito da Atenção Primária de Saúde?”. A coleta de dados ocorreu entre os meses de setembro e outubro a partir da base de dados da Literatura Latino-Americana e do Caribe e Ciências da Saúde (LILACS), Bases de Dados em Enfermagem (BDENF) e Scientific Electronic Library Online (SciELO). 
Para a estratégia de busca foram utilizados os descritores: pré-estabelecidos pelo Descritores em Ciências da Saúde (DECS), sendo "Educação Interprofissional", "Atenção Primária à Saúde" e "Equipe de Assistência ao Paciente", com isso sendo realizada as seguintes combinações: Educação Interprofissional AND Atenção Primária à Saúde e Equipe de Assistência ao Paciente AND Educação Interprofissional. A amostra selecionada entre tantos disponível resultou em 8 artigos, estes foram analisados e categorizados como descreve Mendes, Silveira \& Galvão, respectivamente: 1) Sobre os desafios da aplicabilidade da educação interprofissional e, 2) Os benefícios da educação interprofissional no âmbito da Atenção Básica.

Os critérios de inclusão adotados foram: I) estudos clínicos, randomizados, experimental e quase-experimental que respondessem à questão norteadora a partir da leitura do título e resumo; II) período de publicação entre os anos de 2016 a 2021; e III) estar nos idiomas português, inglês ou espanhol. Os critérios de exclusão envolveram estudos duplicados e que correspondessem a revisão integrativa, livros, cartas ao editor e artigos de nota prévia. Em seguida, foi selecionado o quantitativo de 8 estudos para compor o corpus de análise de artigos elegíveis.

A busca e seleção dos artigos foram realizadas e inicialmente identificou-se os estudos que apresentavam duplicidade entre as bases de dados pesquisadas e após isso, foi feita a avaliação do título e resumo. Por fim, foi realizada a leitura integral dos artigos identificados como potencialmente elegíveis, sendo incluídos no estudo, aqueles que estavam de acordo com os critérios de inclusão e exclusão.

A Figura 1 apresenta o fluxograma de seleção dos resultados encontrados. Para que se tenha a facilidade de compreensão de cada critério utilizado.

Figura 1: Fluxograma de seleção dos resultados da pesquisa.

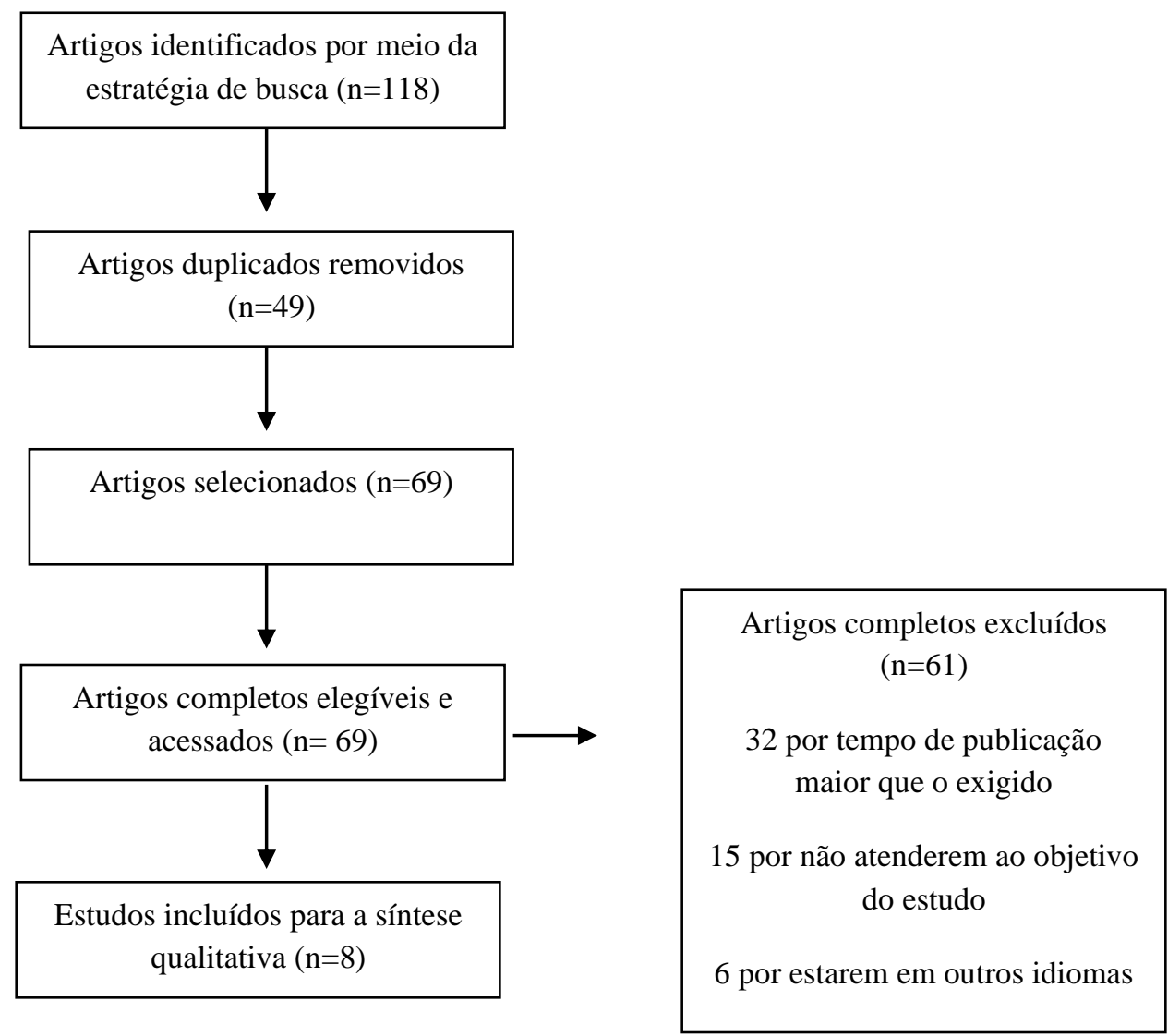

Fonte: Autores (2021). 


\section{Resultados e Discussão}

Após o processo de elegibilidade dos estudos para a concretização da pesquisa de revisão integrativa, foi propicio a leitura na íntegra dos 8 materiais e a caracterização de todos através do corpus de análise representado na Tabela 1, com as informações mais relevantes, como autoria e ano de publicação, título do artigo, objetivo do estudo, delineamento metodológico e resultados. A pesquisa priorizou estudos dos últimos cinco anos, em idiomas português, inglês e espanhol. Dessa forma, possibilitou a coleta de dados literários e informações pertinentes referentes aos desafios na aplicação da EIP nos serviços públicos de saúde, especialmente na atenção primária. Sendo assim, estruturando uma discussão relevante com subcategorias diante a importância do método de educação interprofissional, para integralização e qualificação da assistência em saúde.

A coleta literária compõe artigos do tipo: estudo de caso, qualitativo, quantitativos, revisões, relato de experiência, estudo experimental e entre outros.

Tabela 1. Corpus de análise da pesquisa de revisão integrativa. Brasil, 2021.

\begin{tabular}{|c|c|c|c|c|}
\hline $\begin{array}{l}\text { Autoria e ano de } \\
\text { publicação }\end{array}$ & Título do artigo & Objetivo do estudo & $\begin{array}{c}\text { Delineamento } \\
\text { metodológico }\end{array}$ & Resultados \\
\hline $\begin{array}{l}\text { VENDRUSCULO, } \\
\text { C. et al., } 2020 .\end{array}$ & $\begin{array}{l}\text { Implication of the training } \\
\text { ad continuing education } \\
\text { process for the } \\
\text { interprofessional } \\
\text { performance. }\end{array}$ & $\begin{array}{l}\text { Avaliar as implicações do } \\
\text { processo de formação / } \\
\text { educação permanente dos } \\
\text { profissionais do Núcleo } \\
\text { Ampliado de Saúde da } \\
\text { Família e Atenção Básica } \\
\text { (Nasf-AB) para sua } \\
\text { atuação interprofissional. }\end{array}$ & $\begin{array}{c}\text { Estudo de caso } \\
\text { qualitativo, exploratório } \\
\text { descritivo, realizado } \\
\text { com equipes do Nasf- } \\
\text { AB representativas de } \\
\text { quatro macrorregiões de } \\
\text { saúde do estado de } \\
\text { Santa Catarina. }\end{array}$ & $\begin{array}{c}\text { A formação é fortemente } \\
\text { influenciada pelo Modelo } \\
\text { Biomédico, que atua como um } \\
\text { obstáculo à atuação } \\
\text { interprofissional, frente aos } \\
\text { desafios do cotidiano dos } \\
\text { profissionais do Nasf-AB. A } \\
\text { educação permanente surge } \\
\text { como potencializador para o } \\
\text { sucesso do trabalho } \\
\text { colaborativo entre as equipes, e } \\
\text { entre as equipes e os } \\
\text { profissionais de Saúde da } \\
\text { Família. }\end{array}$ \\
\hline $\begin{array}{l}\text { GRIGGIO, A.P. et } \\
\text { al., } 2020 .\end{array}$ & $\begin{array}{c}\text { Analysis of an } \\
\text { interprofessional } \\
\text { education activity in the } \\
\text { occupational health field }\end{array}$ & $\begin{array}{l}\text { Analisar os resultados de } \\
\text { uma atividade de } \\
\text { Educação } \\
\text { Interprofissional na área } \\
\text { da Saúde do Trabalhador. }\end{array}$ & $\begin{array}{l}\text { Trata-se de uma } \\
\text { pesquisa-ação, que } \\
\text { englobou as etapas de } \\
\text { implementação e } \\
\text { avaliação da } \\
\text { atividade. Foi } \\
\text { desenvolvido em uma } \\
\text { Instituição Pública de } \\
\text { Ensino Superior por } \\
\text { meio de } 15 \text { encontros, } \\
\text { totalizando } 60 \text { horas. }\end{array}$ & $\begin{array}{l}\text { Foram identificadas três } \\
\text { categorias temáticas: I- } \\
\text { Assistência integral; II- } \\
\text { Trabalho como determinante } \\
\text { social do processo saúde- } \\
\text { doença; e III Trabalho em } \\
\text { equipe interprofissional. A } \\
\text { atividade de Educação } \\
\text { Interprofissional foi avaliada } \\
\text { positivamente pelos } \\
\text { participantes, que apontaram as } \\
\text { contribuições dessa estratégia } \\
\text { na construção do conhecimento } \\
\text { direcionado à Saúde do } \\
\text { Trabalhador. }\end{array}$ \\
\hline $\begin{array}{l}\text { TOASSI, R. F. C. } \\
\text { et al., } 2019 .\end{array}$ & $\begin{array}{l}\text { Ensino da graduação em } \\
\text { cenários da atenção } \\
\text { primária: espaço para } \\
\text { aprendizagem } \\
\text { interprofissional }\end{array}$ & $\begin{array}{c}\text { Investigar como o ensino } \\
\text { da graduação em cenário } \\
\text { de prática da Atenção } \\
\text { Primária à Saúde pode } \\
\text { constituir espaço de } \\
\text { aprendizagem } \\
\text { interprofissional, por } \\
\text { meio da análise do } \\
\text { significado de sua } \\
\text { experiência na formação } \\
\text { de futuros profissionais de } \\
\text { saúde. }\end{array}$ & $\begin{array}{l}\text { Pesquisa desenvolvida } \\
\text { em universidade pública } \\
\text { do sul do Brasil, com } \\
\text { dados qualitativos } \\
\text { coletados por } \\
\text { instrumento auto } \\
\text { aplicado online com } \\
\text { estudantes e egressos } \\
\text { (n=186) de } 15 \\
\text { profissões da saúde que } \\
\text { participaram de uma } \\
\text { experiência de educação } \\
\text { interprofissional. }\end{array}$ & $\begin{array}{l}\text { Resultados mostraram que } \\
\text { práticas de ensino } \\
\text { compartilhadas com estudantes, } \\
\text { professores, profissionais da } \\
\text { saúde de diversas áreas e com } \\
\text { usuários, promoveram } \\
\text { momentos de escuta e de } \\
\text { compartilhamento de } \\
\text { experiências, percepções e } \\
\text { diferentes saberes. }\end{array}$ \\
\hline
\end{tabular}




\begin{tabular}{|c|c|c|c|c|}
\hline $\begin{array}{l}\text { AGRELI, H.F. et } \\
a l ., 2019 .\end{array}$ & $\begin{array}{l}\text { Effect of interprofessional } \\
\text { education on teamwork } \\
\text { and on knowledge of } \\
\text { chronic conditions } \\
\text { management }\end{array}$ & $\begin{array}{c}\text { Avaliar o efeito da } \\
\text { educação interprofissional } \\
\text { no clima das equipes de } \\
\text { Atenção Básica à Saúde e } \\
\text { na aquisição de } \\
\text { conhecimentos sobre o } \\
\text { manejo das doenças } \\
\text { crônicas não } \\
\text { transmissíveis. }\end{array}$ & $\begin{array}{l}\text { Estudo quase } \\
\text { experimental de } \\
\text { intervenção em } \\
\text { educação } \\
\text { interprofissional. }\end{array}$ & $\begin{array}{l}\text { Na análise do conhecimento } \\
\text { sobre o manejo das condições } \\
\text { crônicas, as equipes que } \\
\text { participaram da intervenção de } \\
\text { educação interprofissional } \\
\text { apresentaram maior aumento } \\
\text { médio pós-intervenção do que } \\
\text { as equipes do grupo controle (p } \\
<0,001) \text {. Porém, na análise de } \\
\text { ambos os grupos, não houve } \\
\text { variação significativa nos } \\
\text { escores do clima de trabalho } \\
\text { em equipe }(0,061) \text {. }\end{array}$ \\
\hline $\begin{array}{l}\text { PREVIATO, G.F. } \\
\text { et al., } 2018 .\end{array}$ & $\begin{array}{l}\text { A comunicação na } \\
\text { perspectiva dialógica da } \\
\text { prática interprofissional } \\
\text { colaborativa em saúde na } \\
\text { Atenção Primária à Saúde }\end{array}$ & $\begin{array}{l}\text { O objetivo deste estudo } \\
\text { foi analisar a } \\
\text { comunicação enquanto } \\
\text { domínio da prática } \\
\text { interprofissional } \\
\text { colaborativa em Saúde no } \\
\text { processo de trabalho das } \\
\text { equipes da Atenção } \\
\text { Primária à Saúde }\end{array}$ & $\begin{array}{c}\text { Estudo com abordagem } \\
\text { qualitativa, de caráter } \\
\text { descritivo e } \\
\text { interpretativo. }\end{array}$ & $\begin{array}{l}\text { Levantaram-se cinco categorias } \\
\text { de léxicos que, quando } \\
\text { agrupados, revelaram como } \\
\text { ocorre a comunicação entre as } \\
\text { equipes da Atenção Primária à } \\
\text { Saúde. A comunicação, de } \\
\text { caráter interprofissional e } \\
\text { colaborativo, ainda é um } \\
\text { desafio para as equipes de } \\
\text { saúde conduzirem um processo } \\
\text { de trabalho compartilhado, } \\
\text { dialógico e transformador. }\end{array}$ \\
\hline $\begin{array}{l}\text { ESCALDA, P; } \\
\text { PEREIRA, C. M. S. } \\
\text { F. P., } 2018 .\end{array}$ & $\begin{array}{c}\text { Dimensões do trabalho } \\
\text { interprofissional e práticas } \\
\text { colaborativas } \\
\text { desenvolvidas em uma } \\
\text { Unidade Básica de Saúde, } \\
\text { por equipe de saúde da } \\
\text { família. }\end{array}$ & $\begin{array}{l}\text { Identificar as dimensões } \\
\text { do trabalho } \\
\text { interprofissional e das } \\
\text { práticas colaborativas } \\
\text { desenvolvidas por uma } \\
\text { equipe de saúde da } \\
\text { família em uma Unidade } \\
\text { Básica de Saúde. }\end{array}$ & $\begin{array}{c}\text { Pesquisa qualitativa } \\
\text { realizada mediante } \\
\text { observação participante } \\
\text { do trabalho } \\
\text { desenvolvido por } \\
\text { profissionais de saúde. }\end{array}$ & $\begin{array}{l}\text { O estudo realizado evidenciou } \\
\text { avanços na incorporação de } \\
\text { práticas colaborativas no } \\
\text { âmbito da atenção primária e } \\
\text { na criação de espaços mais } \\
\text { favoráveis ao diálogo e ao } \\
\text { estabelecimento de consensos } \\
\text { que resultam em cuidado } \\
\text { integral e segurança do } \\
\text { paciente, a despeito dos } \\
\text { conflitos e das tensões próprias } \\
\text { do processo de trabalho em } \\
\text { saúde. } \\
\end{array}$ \\
\hline $\begin{array}{l}\text { PREVIATO, G. F.; } \\
\text { BALDISSERA, V. } \\
\text { D. A., } 2017 .\end{array}$ & $\begin{array}{l}\text { Domains and } \\
\text { competencies of the } \\
\text { collaborative } \\
\text { interprofission al practice } \\
\text { in primary health care } \\
\text { teams. }\end{array}$ & $\begin{array}{l}\text { Analisar os domínios e } \\
\text { competências da Prática } \\
\text { Interprofissional } \\
\text { Colaborativa em Saúde no } \\
\text { processo de trabalho das } \\
\text { equipes de Estratégia } \\
\text { Saúde da Família e } \\
\text { Núcleo de Apoio à Saúde } \\
\text { da Família. }\end{array}$ & $\begin{array}{l}\text { Estudo exploratório, } \\
\text { descritivo, de } \\
\text { abordagem qualitativa. }\end{array}$ & $\begin{array}{l}\text { Contribuir na compreensão de } \\
\text { como as equipes atuantes na } \\
\text { Atenção Primária à Saúde } \\
\text { organiza seu processo de } \\
\text { trabalho pautado em domínios } \\
\text { e competência para uma Prática } \\
\text { Interprofissional Colaborativa } \\
\text { em Saúde. }\end{array}$ \\
\hline $\begin{array}{l}\text { CASANOVA, I.A. } \\
\text { et al., } 2018 .\end{array}$ & $\begin{array}{c}\text { Educação } \\
\text { interprofissional e a } \\
\text { prática compartilhada em } \\
\text { programas de residência } \\
\text { multiprofissional em } \\
\text { saúde. }\end{array}$ & $\begin{array}{l}\text { Analisar os princípios, } \\
\text { concepções e práticas da } \\
\text { Educação } \\
\text { Interprofissional, com } \\
\text { ênfase na prática } \\
\text { compartilhada em } \\
\text { programas de residência } \\
\text { multiprofissional em } \\
\text { Saúde (PRMS) nas } \\
\text { instituições de ensino } \\
\text { superior (IES) do Estado } \\
\text { de São Paulo, Brasil. }\end{array}$ & $\begin{array}{l}\text { Estudo quali- } \\
\text { quantitativa de caráter } \\
\text { exploratório descritivo. }\end{array}$ & $\begin{array}{l}\text { Os resultados do instrumento } \\
\text { Likert evidenciam satisfação } \\
\text { dos residentes com a formação } \\
\text { para prática colaborativa. Na } \\
\text { perspectiva dos residentes, os } \\
\text { PRMS ampliam e melhoram os } \\
\text { resultados em saúde, } \\
\text { favorecem a atuação centrada } \\
\text { no paciente, a identificação das } \\
\text { necessidades de saúde e o } \\
\text { compartilhamento de práticas e } \\
\text { procedimentos. }\end{array}$ \\
\hline
\end{tabular}

Fonte: Autores (2021). 
Ao avaliar os artigos foram vistas diversas vertentes sobre a prática da EIP nos serviços de saúde, obstáculos que dificultam sua aplicação. Contudo, também foram identificados vários elementos significativos para a concretização da educação interprofissional, mas, os principais foram agrupados na Figura 2 a seguir:

Figura 2. Práticas imprescindíveis para a implementação da educação interprofissional. Brasil, 2021.

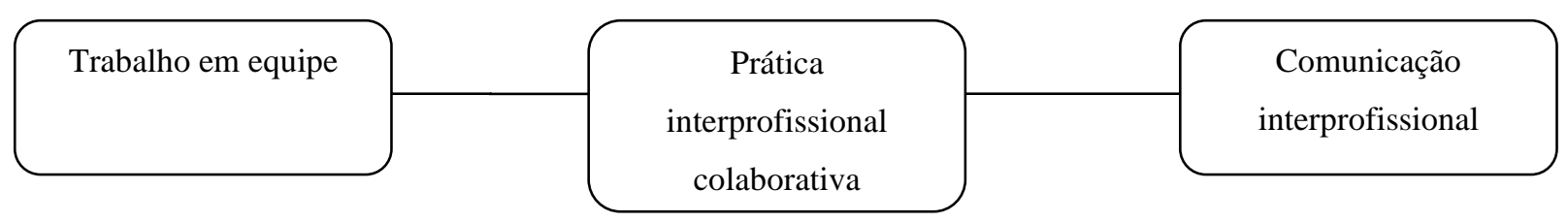

Fonte: Autores (2021).

\section{Sobre os desafios da aplicabilidade da educação interprofissional}

Em um estudo de caso exploratório descritivo com profissionais de saúde percebeu que o trabalho dos profissionais ainda é fortemente voltado para o cuidado individual e curativo, e deixa muito a desejar quanto às orientações para as práticas preventivas e de promoção da saúde. Para os profissionais do Núcleo Ampliado de Saúde da Família e Atenção Básica (NASFAB), apesar das mudanças em algumas profissões, essas marcas ainda são notórias nas atitudes da equipe e gestores. Por isso, às vezes se sentem invisíveis no contexto da saúde pública / coletiva e da atenção básica (Vendruscolo, Trindade, Maffissoni, Martini, Filho \& Sandri, 2020).

Os profissionais de saúde do NASF revelam que o processo de formação na graduação possui um déficit de conteúdos e práticas referentes à atuação generalista na atenção básica com algumas variações de um curso para outro ou entre universidades. Revelando que há uma necessidade de ações e encontros de educação permanente no cotidiano de trabalho. E ainda ressaltam o diferencial de atuação dos profissionais que participaram de residência multiprofissional, visto que são mais preparados para uma formação voltada ao SUS (Sistema Único de Saúde) (Vendruscolo, Trindade, Maffissoni, Martini, Filho $\&$ Sandri, 2020).

Sendo perceptível que um dos obstáculos para a prática de EIP nos serviços, é fruto de uma formação individual, curativista e um modelo hospitalocêntrico, ou seja, que diverge da realidade dos sujeitos, serviço-comunidade e suas demandas. E com essas diferenças na atuação, comprometem a assistência e integralização dos serviços no SUS. Uma vez que, as formações não se comunicam entre si, desde a graduação, fazendo com que na prática profissional haja um déficit na comunicação interprofissional e consequentemente a falta da interprofissionalidade em saúde.

Depoimentos apontaram diversos desafios na rotina de trabalho para a consolidação do modelo de atenção integral em saúde, salientando influências do modelo biomédico nos processos de trabalho e nas relações interprofissionais. Foi listado o despreparo profissional em compreender e atender as demandas dos trabalhadores para além da doença; erros no sistema de referência e contra-referência; compreensão da comunidade e dos profissionais á respeito da promoção da saúde; falta de investimentos em capacitação dos profissionais, sobrecarga do serviço e a escassez de recursos físicos e materiais (Griggio, Silva, Rossit, Mieiro, Miranda \& Miniel, 2020).

O despreparo profissional está relacionado com a falta de contato com as problematizações nos serviços de saúde desde a graduação e também a falta de uma comunicação interprofissional, tornando-se assim, limitada as ações e intervenções, desconsiderando os determinantes do processo de saúde-adoecimento; a falha no sistema de referência e contra-referência diz muito sobre a comunicação nos serviços de saúde e como os profissionais se portam diante da integralidade da assistência; também é perceptível a escassez do conhecimento sobre o território e população abrangente, o que consequentemente acarreta a falta de promoção de saúde; E ainda vale lembrar, a sobrecarga desses profissionais e a ausência de investimento local para 
capacitações e educação permanente, que são essenciais para o aprimoramento do fazer educação interprofissional. Dessa forma, esses desafios perpetuam durante a prática profissional e o trabalho colaborativo se torna inexistente, levando em conta que os profissionais já presentes nos serviços possuem uma formação individualista, no entanto, isso precisa ser desconstruído para que a prática colaborativa esteja presente e que a interprofissionalidade seja a ferramenta principal para a qualificação da assistência.

Pesquisas e indicadores mostram que a EIP possibilita o aumento de conhecimentos, atitudes, habilidades e comportamentos que auxiliam á prática colaborativa em saúde, aperfeiçoamento do trabalho em equipe, desenvolvimento do respeito e o reconhecimento das habilidades dos envolvidos. O conceito de EIP em sua prática proporciona que os profissionais utilizem todo conhecimento e capacidade da sua formação, e a Organização Mundial de Saúde (OMS) tem auxiliado e se esforçado para ajudar os países na implementação, com a organização de eventos á respeito e reuniões, também visa buscar parceiras com o Canadá, Reino Unido, Espanha e Estados Unidos, onde a educação interprofissional já é existente nas graduações em saúde (Vendruscolo, Trindade, Maffissoni, Martini, Filho \& Sandri, 2020).

Essas ações permitem o fortalecimento às oportunidades de atuação efetivas e colaborativas, que são direcionadas as pessoas e comunidades, uma assistência mais holística e eficaz para a resolutividade dos processos de saúde-doença, agregando o trabalho em equipe e a interprofissionalidade como atuantes indispensáveis.

\section{Sobre os benefícios da educação interprofissional no âmbito da Atenção Básica}

Em outro estudo, foram trabalhados com os profissionais atuantes nos serviços, encontros norteados por metodologias ativas de aprendizagem como, problematização, dinâmica de grupo, oficina, simulação, aula invertida, discussão com gatilhos e apresentação de estudos de caso (Griggio, Silva, Rossit, Mieiro, Miranda \& Miniel, 2020). Essas intervenções e capacitações por meio das metologias ativas de aprendizagem permitem a desconstrução do "automático" nos serviços, frente a uma dificuldade, ou seja, uma oportunidade de tentar o novo, o ser junto e o fazer em equipe.

No entanto, quando se reflete sobre novas estratégias para potencializar os serviços de saúde, o profissional é visto como autor principal de mudanças, mas, também é válido que se é pra mudar a prática profissional, que a formação também passe por ajustes, sendo pensadas maneiras de integrar a interprofissionalidade já na instituição de graduação, para que futuros profissionais de saúde possam estar ainda mais aptos para a realidade do SUS, determinantes de saúde e capacitados para o desenvolvimento do trabalho em equipe e colaborativo.

Um dos atributos que compõem a estruturação da experiência de EIP é a participação de estudantes e professores de diferentes profissões, em conjunto, na prática, com a reciprocidade de ideias, experiências, habilidades e conhecimentos. Observou-se que á prática de ensino baseada no conhecimento do território, trabalho em equipe, longitudinalidade e integralidade do cuidado, promoveu aprendizagens com caráter interprofissional na graduação, os quais possuem relevantes potências para fomentar a colaboração no trabalho em equipe e a atenção integral à saúde (Toassi, Olsson, Lewgoy, Bueno \& Peduzzi, 2019).

A literatura aponta indícios de impactos positivos da educação interprofissional nas atuações, conhecimento específico e percepções interprofissionais. Entretanto, o trabalho colaborativo e o clima de trabalho em equipe, incluem aspectos relacionais, contextuais, organizacionais e algumas tensões, bem como competências colaborativas versus autonomia de cada profissão, essas tensões podem ser desafiadoras, em principal, num curto espaço de tempo. Essas tensões presentes na prática não necessariamente precisam ser cessadas de vez, mas, ao menos enfrentadas de forma que não interfira no avanço do serviço em prol da prática colaborativa (Agreli, Peduzzi, Silva, Mascarelle \& Espinoza, 2019).

No dia a dia do trabalho em saúde, há tensões, conflitos e discordâncias com relação à condução ou à responsabilização de casos, à tomada de decisão ou às escolhas de procedimentos e terapêuticas que precisam ser contornados ou superadas para 
que se haja o trabalho colaborativo. O modo como tais tensões ou conflitos são percebidos ou mediados poderá contribuir para maior ou menor interação da equipe (Escalda \& Parreira, 2018).

Sendo assim, percebe-se que a prática de EIP sempre terá algum desafio a ser enfrentado, seja diante da realidade, dos profissionais ou até mesmo na comunicação, porém, esses obstáculos não podem interferir na prática colaborativa e os profissionais precisam manter a comunicação interprofissional e chegar a uma solução de forma que seja ideal e melhor para todos os sujeitos envolvidos no processo, visando não interferir na assistência ao indivíduo, serviço ou comunidade.

A comunicação interprofissional requer uma conversação solidária, que fomentem e reflitam as transformações no agir, visadas á promoção da humanização, da criação e da libertação dos processos de trabalho permutantes. A construção de um projeto terapêutico singular, construído a partir de uma discussão entre usuário e equipe interprofissional, é um método de conseguir agregar a interprofissionalidade em prol do indivíduo ou população (Previlato \& Baldissera, 2018).

Para a reciprocidade coletiva, é necessário saber manter a comunicação interprofissional, a troca de informações ou a soma de soluções, o desenvolvimento de novas estratégias para contribuir em superar as dificuldades, porém, também deve ser saber o que é a competência de determinada área. Nesse contexto, há muitas controvérsias, pois, como se faz junto, se aprende separadamente? E ai se estabelece a precisão da desconstrução de modelo biomédico.

Um estudo realizado com residentes multiprofissionais evidenciou a necessidade de uma definição conjunta de indicadores que avaliam a qualidade do serviço prestado pela equipe, visando melhorar o cuidado ao paciente. A prática colaborativa proporciona a relação entre eles; permite que cada profissão tenha não só uma compreensão mais profunda de seu próprio desempenho, mas também complemente e potencialize o de outros profissionais. (Casanova, Batista \& Moreno, 2018). Esse contato com a problematização na residência permite que o indivíduo desenvolva estratégias e habilidades que contribuam com o perfil de educação interprofissional e quando esse contato não acontece e profissionais de saúde atuantes lhe dão com muitas dificuldades em realizar a mudança do agir em conjunto (Casanova, Batista \& Moreno, 2018).

Dessa forma, o presente estudo visou apresentar os desafios que estão relacionados à prática da educação interprofissional, resgatando desde formação dos profissionais até sua atuação no SUS. Foi evidenciado que se precisa de mudanças na formação de novos profissionais e aos já existentes, uma educação permanente, para que assim, seja possível estratégias interprofissionais e a melhoria na assistência em saúde.

\section{Conclusão}

Assim, mediante a todos os desafios o desenvolvimento da educação interprofissional e interdisciplinar só será consolidada quando for ampliada, disseminada e potencializada as experiências educacionais para que, se tenha interações entre as variadas áreas para que seja visto o impacto dessas ações na Atenção Básica de Saúde. Com isso, é preciso que as diversas áreas colaborativas reconheçam os distintos coletivos de pensamento para que se tenha uma investigação e problematização das racionalidades com seus devidos valores presentes em cada discurso e prática realizada.

Dessa forma, os diferentes estilos de pensamento são socialmente construídos ampliam as possibilidades de que os profissionais trabalhando de forma interprofissional, tornando-se mais conscientes de seu papel e prática na atenção à saúde. Contudo, percebe-se que ainda existem empecilhos na absorção das práticas interprofissionais, pois existem recusas na adoção de práticas colaborativas, devido à ausência de qualificação e capacitação sobre determinada abordagem e conteúdo. Assim, faz-se necessário a ampliação do acesso aos trabalhadores da área da saúde as ações que estimulem a interprofissionalidade, principalmente aqueles alocados na atenção básica. 


\section{Referências}

Agreli, H.F., Peduzzi, M., Silva, M. C., Mascarelle, R. C. V., \& Espinoza, P. (2019). Effect of interprofessional education on teamwork and on knowledge of chronic conditions management. Revista latino Americana de 27:e3203.https://www.scielo.br/j/rlae/a/x38MRGkmVNDrNwt4R5xdfLF/?lang=pt\&format=pdf

Bourgeault, I. L., Grignon, M., \& Grignon, M. L. (2013). A comparison of the regulation of health professional boundaries across OECD countries. Eur $J$ Comp Econ. 10(2), 199-223. https://ideas.repec.org/a/liu/liucej/v10y2013i2p199-223.html

Cardoso, C. G. \& Hennington, E. A. (2011). Trabalho em equipe e reuniões multiprofissionais de saúde: uma construção à espera pelos sujeitos da mudança. Trab Educ Saude. 9(1), 85-112. https://www.scielo.br/j/tes/a/YS6JT5hmc8YtsgGhfFYzN5S/abstract/?lang=pt

Casanova, I. A., Batista, N. A. \& Moreno, L.R. (2018). Interprofessional Education and shared practice in multiprofessional health residency programs. Interface. 22. https://www.scielo.br/j/icse/a/cPBjVyTv9xfrP7NndsRG8pB/abstract/?lang=pt

Ceccim, R. B. (2018). Conexões e fronteiras da interprofissionalidade: forma e formação. Interface comunicação, saúde e educação. 22(2), 1739-1749. https://www.scielo.br/j/icse/a/XRJVNsRHcqfsRXLZ7RMxCks/abstract/?lang=pt

Cerballos, A. G. C. (2015). Determinação social do processo saúde e doença. Modelos conceituais de saúde, determinação social do processo saúde e doença, promoção da saúde. Recife: Grupo saber tecnologias educacionais e sociais UNA-SUS UFPE, 1-20. https://ares.unasus.gov.br/acervo/html/ARES/3332/1/2mod_conc_saude_2016.pdf.

Costa, M. V., Filho, J. R. F., Brandão, C., \& Silva, J. A. M. (2018). A educação e o trabalho interprofissional alinhados ao compromisso histórico de fortalecimento e consolidação do Sistema Único de Saúde. Interface. 22. https://www.scielo.br/j/icse/a/FrQWDLv8Tk8bQYXcTCpc9GP/?lang=en

Escalda, P. \& Parreira C. M. S. F. (2018). Dimensions of interprofessional work and of collaborative practices developed at a primary care unit by a Family Health team. Interface. 22.https://www.scielo.br/j/icse/a/Kg5M3YCMBKsbkx4QF6DJvJt/?lang=en

Griggio, A. P., Silva, J. A. M., Rossit, R. A. S., Mieiro, D. B., Miranda, F. M., \& Miniel, V. A. (2020). Analysis of an interprofessional education activity in the occupational health field. Revista Latino-Americana de Enfermagem. 28. https://www.scielo.br/j/rlae/a/YqkFfkL9PHyvj7MsTnb39wr/?lang=en

Ludke, M. \& Andre, M. E. D. A. (2013). Pesquisas em educação: uma abordagem qualitativa. E.P.U.

Mendes, K. D. S., Silveira, R. C. C. P., \& Galvão, C. M. (2008). Revisão integrativa: método de pesquisa para a incorporação de evidências na saúde e na enfermagem. Texto e contexto - Enfermagem, 17(4), 758-764. https://doi.org/10.1590/S014-07072008000400018.

Peduzzi, M. \& Agreli H. F. (2018). Teamwork and collaborative practice in Primary Health Care. Interface. 22. https://www.scielo.br/j/icse/a/MR86fMrvpMcJFSR7NNWPbqh/abstract/?lang=en

Peduzzi, M. (2016). O SUS é interprofissional. Interface - Comunicação, Saúde, Educação. 20(56), 199-201. https://www.scielo.br/j/icse/a/7MgQL4JM9dRYFDLYYzQVLHM/?lang=pt

Peduzzi, M., Agreli, H. L. F., Silva, J. A. M, \& Souza, H. S. (2018). Trabalho em equipe: uma revisita ao conceito e a seus desdobramentos no trabalho interprofissional. Trabalho, educação e saúde. 18(1). https://www.scielo.br/j/tes/a/RLtz36Ng9sNLHknn6hLBQvr/?lang=pt

Previato, G. F. \& Baldissera, V. D. A. (2017). Domains and competencieis of the collaborative interprofessional practice in primary health care teams. Revista de enfermagem Journal of Nursing UFPE online. DOI:10.5205/reuol.11077-98857-1-SM.1105201729.

Previato, G. F. \& Baldissera, V. D. A. (2018). Communication in the dialogical perspective of collaborative interprofessional practice in Primary Health Care. Interface. 22(2). https://www.scielo.br/j/icse/a/L9VS9vQGQtzPTpyZztf4cJc/?lang=en

Rossit, R. A. S., Freitas, M. A. O., Batista, S. H. S. S., \& Batista, N. A. (2018). Construção da identidade profissional na educação interprofissional em saúde: percepção dos egressos. $\quad$ Interface $\quad$ Comunicação, $\quad$ Saúde $\quad e \quad$ Educação. https://www.scielo.br/j/icse/a/wtqgWTz6VYZjqZW3Gp5yG4F/abstract/?lang=pt

Santana, M. C. C. P. \& Rossit, R. A. S. (2017). Interprofissionalismo nas residências multiprofissionais em saúde: análise na região nordeste do Brasil. Journal of management and primary health care. 8(3), 45-46. DOI: https://doi.org/10.14295/jmphc.v8i3.609.

Silva, J. A. M., Peduzzi, M., Orchad, C., \& Leonello, V. M. (2015). Interprofessional education and collaborative practice in Primary Health Care. Rev Esc Enferm USP. 49(2), 16-24. https://pubmed.ncbi.nlm.nih.gov/26959149/

Toassi, R. F. C., Olsson, T. O., Lewgoy, A. M. B., Bueno, D., \& Peduzzi, M. (2019). Ensino da graduação em cenários da atenção primária: espaço para aprendizagem interprofissional. Trabalho, educação e saúde. https://www.scielo.br/j/tes/a/QsJJqQrDcq5cvqtGz4vhgNb/?lang=pt\&format=pdf

Venduscrolo, C., Tridande, L. L., Maffissoni, A. L., Martini, J. G., Filho, C. C. S., \& Sandri, J. V. A. (2020). Implication of the training ad continuing education process for the interprofessional performance. Revista Brasileira de https://www.scielo.br/j/reben/a/zJkQrDPVcBSH94b3N6c8Scm/?lang=en 